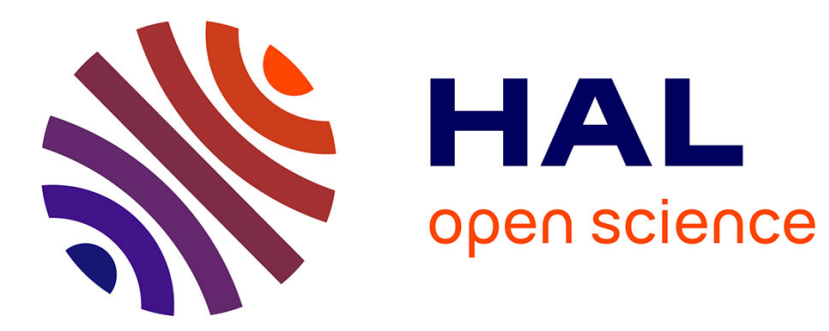

\title{
Mixing mechanism in a two-dimensional bubble column
}

Elise Alméras, Frédéric Risso, Véronique Roig, Cécile Plais, Frédéric Augier

\section{To cite this version:}

Elise Alméras, Frédéric Risso, Véronique Roig, Cécile Plais, Frédéric Augier. Mixing mechanism in a two-dimensional bubble column. Physical Review Fluids, 2018, 3 (7), pp.1-19. 10.1103/PhysRevFluids.3.074307 . hal-02014282

\section{HAL Id: hal-02014282 \\ https://hal-ifp.archives-ouvertes.fr/hal-02014282}

Submitted on 9 Apr 2019

HAL is a multi-disciplinary open access archive for the deposit and dissemination of scientific research documents, whether they are published or not. The documents may come from teaching and research institutions in France or abroad, or from public or private research centers.
L'archive ouverte pluridisciplinaire HAL, est destinée au dépôt et à la diffusion de documents scientifiques de niveau recherche, publiés ou non, émanant des établissements d'enseignement et de recherche français ou étrangers, des laboratoires publics ou privés. 


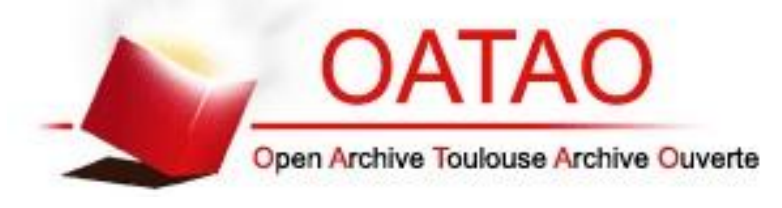

Open Archive Toulouse Archive Ouverte

OATAO is an open access repository that collects the work of Toulouse researchers and makes it freely available over the web where possible

This is an author's version published in: http://oatao.univ-toulouse.fr/22904

\section{Official URL:}

https://doi.org/10.1103/PhysRevFluids.3.074307

\section{To cite this version:}

Alméras, Élise and Risso, Frédéric and Roig, Véronique and Plais, Cécile and Augier, Frédéric Mixing mechanism in a twodimensional bubble column. (2018) Physical Review Fluids, 3 (7). 1. ISSN 2469-990X

Any correspondence concerning this service should be sent to the repository administrator: tech-oatao@listes-diff.inp-toulouse.fr 


\title{
Mixing mechanism in a two-dimensional bubble column
}

\author{
Elise Alméras* \\ Institut de Mécanique des Fluides de Toulouse, IMFT, Université de Toulouse, CNRS, Toulouse, France \\ and IFP Energies nouvelles, Rond-point de l'échangeur de Solaize, BP 3, 69360 Solaize, France \\ Frédéric Risso and Véronique Roig \\ Institut de Mécanique des Fluides de Toulouse, IMFT, Université de Toulouse, CNRS, Toulouse, France \\ Cécile Plais and Frédéric Augier \\ IFP Energies nouvelles, Rond-point de l'échangeur de Solaize, BP 3, 69360 Solaize, France
}

(Received 21 June 2017; published 31 July 2018)

\begin{abstract}
The present contribution investigates the mixing of a passive scalar by a homogeneous bidimensional bubble swarm rising at high Reynolds number in a liquid initially at rest. Mixing experiments are performed in a Hele-Shaw cell for gas volume fractions $\alpha$ ranging from $3.0 \%$ to $14.0 \%$. A weakly diffusive passive dye is injected within the swarm, and the temporal evolution of the spatial distribution of concentration is measured. The vertical distribution of concentration shows an upward propagation and a spreading due to the mixing induced by the unsteady open wakes of the bubbles. A one-dimensional large-scale model involving an intermittent and convective mechanism has been developed to describe the global evolution of the concentration distribution in the vertical direction. Based on experimental observations, it assumes that each bubble catches a given volume of fluid $V_{t}$ in its wake and transports it over a certain length $L$ before releasing it. A good agreement is found between the experimental concentration profiles measured in the vertical direction and the model prediction. The comparison between the model and the experiments allows the determination of the transported volume $V_{t}$ and the transport length $L$ as a function of the gas volume fraction. It appears that the transported volume is related to the characteristic length of the velocity deficit at the rear of the bubble. The transport length, which is related to the correlation length of the dye patches, shows two regimes. At low gas volume fraction, it is controlled by the viscous length related to the flow damping at the walls, whereas, at higher gas volume fraction, it is limited by the distance between two successive bubbles. The mixing properties are finally characterized from the first three-order moments of the dye distribution, which are determined by means of the model. The upward propagation of the dye is shown to scale as $\alpha V$, where $V$ is the bubble rise velocity. The spreading of the concentration distribution is characterized by an effective diffusivity, which presents strong similarities with the diffusion coefficient measured in a three-dimensional bubble column [Alméras et al. J. Fluid Mech. 776, 458 (2015)]. At low gas volume fraction, it increases as $\sqrt{\alpha}$, whereas it saturates at high gas volume fraction. The dye distribution also is asymmetric with a significant skewness coefficient which slowly decreases in time. Therefore, the dye transport cannot be described as a purely diffusive process over the time required for the dye to spread over the cell.
\end{abstract}

DOI: 10.1103/PhysRevFluids.3.074307

*elise.almeras@ensiacet.fr 


\section{INTRODUCTION}

Bubble columns are commonly used for chemical and biological processes because it is the simplest way to bring two phases into contact without any mechanical part. Most technologies involve large tanks where bubbles are rising at high Reynolds number in more or less dense bubbly flows operated in homogeneous or heterogeneous regimes. Other technologies originating from nuclear engineering design use confined bubble columns and may also be envisaged for chemical processes since they ensure efficient mixing (Willmarth and Ishii [1], Spicka et al. [2]). Even if two- and three-dimensional bubble columns share some similarities, different flow structures are developing within the liquid phase due to the presence of confinement. In particular, the shear induced by the walls attenuates the flow disturbances generated by the bubbles and prevents the development of turbulence. Depending on the bubble column geometry, various mixing mechanisms can thus be observed, resulting in contrasted mixing properties. In general, considering passive scalar transport in bubbly flow, four mechanisms can be distinguished, which are the mixing by (1) primary closed wakes, (2) secondary open wakes, (3) irrotational flows around the compound made by the bubbles and their primary closed wake, and (4) turbulent fluctuations. Depending on the flow configuration, mixing results from a different combination of these mechanisms.

In a homogeneous three-dimensional bubble column, the mixing of a passive scalar results from the total agitation, which combines the turbulent fluctuations and the transport by the wakes (Alméras et al. [3]). In this case, mixing can be described by a regular diffusive process, which allows one to model it by an effective diffusivity. The effective diffusivity can be used as a subgrid model in numerical simulations (Alméras et al. [4]) and presents two main properties. First, it is anisotropic since mixing is more efficient in the vertical direction than in the horizontal one. This anisotropy is the signature of the anisotropy of the turbulent velocity fluctuations. Second, the diffusivity saturates when the gas volume fraction is increased. More generally, the diffusion coefficient can be modeled as the product of the variance of the velocity fluctuations by a timescale which is either the integral timescale of the turbulence at low gas volume fraction or the average time between two consecutive bubbles at large gas volume fraction.

In a Hele-Shaw cell, the flow is confined so that no turbulence can develop because of the shear stress at walls (Bouche et al. [5]). Therefore, this experimental configuration allows one to get a better understanding of the contribution of the wakes and of the irrotational flow to the mixing. Eames and Bush studied the mixing induced by the irrotational flow around the compound made by a cylindrical bubble and its close primary wake (Eames and Bush [6,7]). For that purpose, the mixing induced by a single large bubble with a stable trajectory was studied. A model, based on the drift volume displaced by the potential flow around the compound made by the bubble and the stable closed wake, was then developed for a homogeneous bubble swarm. It introduces an equivalent longitudinal diffusivity $D=C_{m}^{\prime}(1+\epsilon) \alpha V L_{p}$ [where $C_{m}^{\prime}$ is the added-mass coefficient of the compound body, $\alpha$ is the gas volume fraction, $(1+\epsilon) \alpha$ is the volume fraction of the compound body, $V$ the bubble rise velocity, and $L_{p}$ the characteristic length of the longitudinal displacement of the drift volume]. As already noticed by Eames and Bush [6], the role of secondary open wakes may be important and limits the prediction by irrotational approaches. The mixing induced by the secondary open wakes has been experimentally characterized in a homogeneous confined bubbly flow with unstable bubble wakes and vortex shedding (Bouche et al. [8], Alméras et al. [9]). In these works, mixing was found to be incompatible with a purely diffusive process and strongly related to successive random intermittent transport in the vicinity of the bubbles. Two characteristic timescales could be distinguished in this flow configuration. The first one describes the global decrease of the concentration, which lasts approximately $10 \mathrm{sec}$. The second one characterizes random fluctuations that are of the order of a few tenths of a second. Schmidt and co-workers introduced a stochastic model to describe such an intermittent mixing mechanism, valid when dispersion is dominated by the transport due to entrainment in bubble wakes (Schmidt et al. [10,11], Nassar et al. [12]). They first developed a model where liquid randomly travels, either in the wakes at a velocity related to wake entrainment or in the interstitial region between the wakes at the average velocity of this zone. 


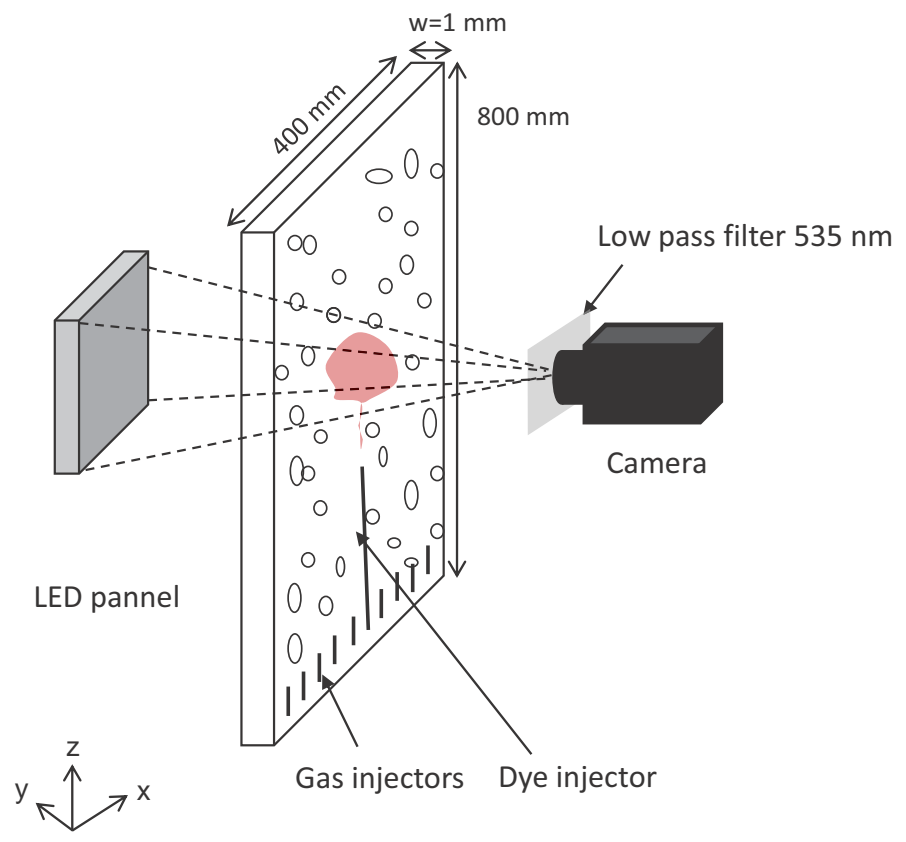

FIG. 1. Experimental setup.

Then they extended their model by defining the probability for each fluid particle to be in each of these two regions. Their proposal was surprisingly not taken over in literature to our knowledge. An explanation may be found in difficulties arising in applying their model, which requires estimating the statistical properties of both random fields.

The aim of the present work is to describe the global evolution of the concentration field and to propose a theoretical model for the transport by the open secondary wakes. Fluctuations of concentration will not be analyzed in detail. In the present work, experimental investigations are performed in flow conditions similar to those of Bouche et al. [8] and Alméras et al. [9] in order to reveal the underlying mixing mechanism. The present measurement method relies on high-frequency visualization of the light absorption by a fluorescent dye over a wide region of the flow, which provides a rather complete description of the temporal evolution of the concentration field $C(\mathbf{x}, t)$. By combining these measurements with an original numerical large-scale model, a description of the statistical distribution of passive scalar transported in a confined bubble swarm can be achieved.

The paper is organized as follows. Section II describes the experimental configuration and the measurement technique. Section III presents the experimental results. Section IV describes the numerical model, which is compared to the experiments in Sec. V. In Sec. VI a physical interpretation of the model parameters is proposed. The centered moments of the concentration distribution obtained due to the model are discussed in Sec. VII. Concluding remarks are proposed in Sec. VIII.

\section{EXPERIMENTAL SETUP AND MEASUREMENT TECHNIQUE}

Experiments were carried out to study the mixing of a passive dye by a homogeneous swarm of bubbles rising in a Hele-Shaw cell (Fig. 1). The setup has already been described in previous publications (Bouche et al. [5,13] and Alméras et al. [9]). It is composed of two glass plates $400 \mathrm{~mm}$ wide and $H=800 \mathrm{~mm}$ high, separated by a gap $w$ of $1 \mathrm{~mm}$. The tank is filled with distilled water in which magnesium salt $\left(\mathrm{MgSO}_{4}\right)$ at a concentration of $5 \times 10^{-2} \mathrm{~mol} / \mathrm{l}$ is dissolved. The salt solution is first mixed by a magnetic stirrer and then filtered to remove solid residuals. Adding a small amount of salt inhibits coalescence without significantly changing the physical properties of the fluids. Under 
this condition a weak contamination of the interface is likely. However, it will not affect significantly the bubble dynamics owing to the short residence time of the bubble in the column and the fact that we are considering a high-Reynolds-number flow regime far beyond the onset of bubble wake instability and oscillatory path. The liquid phase is initially at rest, and bubbles are generated at the bottom of the cell due to capillary tubes of inner diameter $0.6 \mathrm{~mm}$. The capillary tubes are regularly distributed so that the bubbles distribution is homogeneous. A sufficiently large gas tank supplies the capillary tubes in order to get a stationary gas flow rate. The gas volume fraction $\alpha$ is varied from $3.0 \%$ to $14.0 \%$. In this range of $\alpha$, the averaged equivalent diameter $d$ of the in-plane projected area of the bubbles evolves from 4.0 to $4.6 \mathrm{~mm}$, and the bubble population presents a weak dispersion in diameter (Bouche et al. [13]). As the bubble diameter is large compared to the width of the gap $(d / w \geqslant 1)$, bubbles are flattened with ellipsoidal in-plane projected shapes. The bubbles are not in contact with the walls, and a thin liquid film is always present at the walls. The Archimedes number $\mathrm{Ar}=\sqrt{g d} d / v$ is larger than 650 (where $g$ is the gravity acceleration and $v$ the kinematic viscosity) and the Bond number Bo $=\rho g d^{2} / \sigma$ is less than 1.7 (where $\sigma$ is the surface tension). Whatever the gas volume fraction, the Reynolds number of the bubbles $\operatorname{Re}=V_{s b} d / v$ is in the range 370-630 (where $V_{s b}$ is the rising velocity of a single bubble). As $\operatorname{Re} \gg 1$ and $\operatorname{Re}(w / d)^{2} \gg 1$, the flow is dominated by inertia. When isolated, such bubbles have a periodic motion related to the instability of their wake and present very weak oscillations of their shape (Roig et al. [14]). In the confined bubble swarm, bubbles show similar ellipsoidal shapes and unsteady motions. The mean velocity $V$ of the bubbles in the swarm is slightly modified as compared to the rising velocity $V_{s b}$ of a single bubble, which is about $10 \mathrm{~cm} / \mathrm{s}$ : the normalized vertical mean velocity $V / V_{s b}$ increases from 1 to 1.2 as the gas volume fraction increases in the explored range (Bouche et al. [13]). Previous investigations of the liquid motions induced by an isolated bubble or a bubble swarm indicate that, except in the vicinity of the curved interfaces of the bubbles, the flow induced by their rising motion can essentially be considered as parallel to the plates. The perturbation of the liquid induced by an isolated bubble is characterized by an unstable wake consisting of a vertical entrainment in the vicinity of the bubble and a periodic release of in-plane vortices with axes perpendicular to the walls (Roig et al. [14], Filella et al. [15]). The motion induced in the liquid is quickly dampened by the shear stress at the walls. It decreases exponentially with a characteristic distance $\delta$ proportional to the viscous length $l_{v} \approx V w^{2} / \nu$. Bouche et al. [5] have shown that in a confined bubble swarm liquid fluctuations thus result from disturbances localized near the bubbles and direct interactions between them. Due to confinement, released vortices cannot diffuse or interact deeply in a nonlinear way before they are dissipated. The liquid agitation is random, but turbulence cannot develop.

Mixing experiments consist in injecting a passive fluorescent dye (Rhodamine WT) at high Schmidt number ( $\mathrm{Sc}=v / D_{m} \approx 4000$ where $D_{m}$ is the molecular diffusivity) at an initial concentration $c_{0}=2 \times 10^{-3} \mathrm{~mol} / 1$, due to a long capillary tube of inner diameter $0.6 \mathrm{~mm}$ (Fig. 1). The injection point is located $400 \mathrm{~mm}$ above the gas injectors, in a region where the bubbles are homogeneously distributed and have already reached their terminal velocity. A volume of $1 \mathrm{ml}$ of rhodamine is injected during $2 \mathrm{~s}$ by using a syringe pump. During the injection, a jet is generated at the tip of the capillary tube, but it is attenuated in approximately one viscous time $T_{v}=w^{2} / v=1 \mathrm{~s}$. In the present study, the mixing is studied from $2 \mathrm{~s}$ after the end of the dye injection to ensure that the liquid flow generated by the injection has vanished. The time at which we start studying the mixing induced by the bubbles is denoted $t_{0}$ thereafter; it thus corresponds to $4 \mathrm{~s}$ after the start of the dye injection. For each gas volume fraction studied in the present paper, five mixing experiments have been performed to check the reproducibility of the experimental results.

Measurement techniques based on laser-induced fluorescence have already been developed to study mixing in a thin-gap cell. They were, however, limited to low-frequency measurements of an extended concentration field (Bouche et al. [8]) or to high-frequency measurement at a given point (Alméras et al. [9]). In this study, we use a measurement technique based on the absorption of the white light emitted by a LED panel since this allows us to measure large concentration fields at high frequency without the need of correcting light reflections on bubble interfaces as in other techniques. 


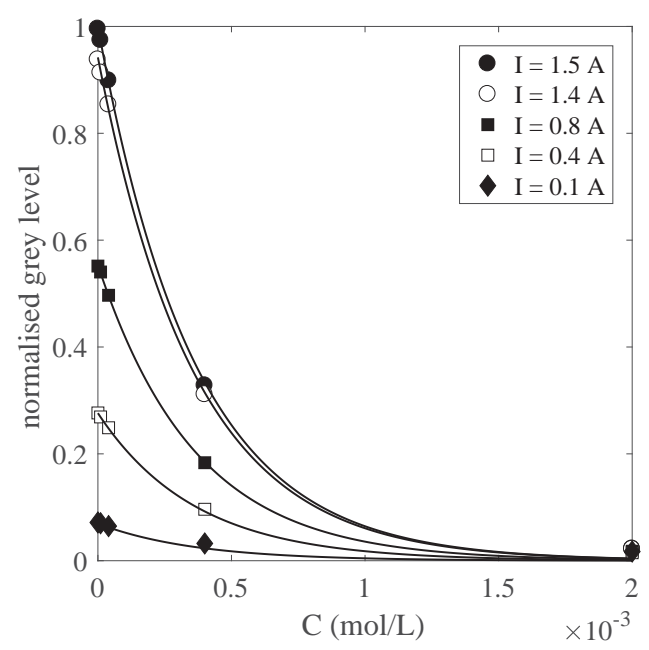

FIG. 2. Calibration curves for different powers of the LED panel (defined by the electricity supply intensity $I$ ). The symbols show the measured gray level as a function of the concentration, the lines the calibration relation given by Eq. (1).

The field of concentration of the dye is visualized by means of the imaging system described in Fig. 1. The measurement is based on the absorption by the fluorescent dye of the light emitted by a white LED panel (PHLOX SLLUB) located on one side of the cell. On the other side, a camera (DIMAX S4, PCO) with a 105-mm lens images a field of $148 \times 150 \mathrm{~mm}^{2}$ at an acquisition frequency of $250 \mathrm{~Hz}$. In order to film only the light absorbed by the fluorescent dye, a notch filter of wavelength $532 \mathrm{~nm}$ (Lavision, ref. VZ07 -0058/80) is set in front of the camera lens. Calibration is required to convert the gray level of each pixel into a concentration. Calibration tests are performed by filling the cell with homogeneous solutions of rhodamine WT at known concentrations. A first calibration has been done by varying the concentration of the rhodamine WT. A second one by changing the intensity of the light emitted by the LED panel. Symbols in Fig. 2 show the calibration for five different intensities of the LED panel. At a given LED panel intensity $I$, the gray levels $G L$ decrease exponentially with the concentration $C$. We can hence write the following calibration relation:

$$
G L(C)=G L(C=0) \exp \left(-\frac{C}{\epsilon}\right)
$$

where $\epsilon$ is the absorption coefficient, which is independent of the LED panel intensity and equal to $3.6 \times 10^{-4} \mathrm{~mol} / \mathrm{l}$. This relation is in agreement with Beer-Lambert law of absorption. Equation (1) has been superimposed to the experimental data for the five LED panel intensities in Fig. 2. Equation (1) fits well the experimental data for all the LED panel intensities. This relation will therefore be used to convert gray levels into concentrations. Before converting gray levels into concentrations, a treatment is applied to the raw images $I_{r}$. First, the inhomogeneities of the lighting by the LED panel are corrected by using the following relation:

$$
I_{c}=\frac{I_{r}}{I_{b}} \times \operatorname{mean}\left(I_{b}\right),
$$

where $I_{c}$ is the corrected image and $I_{b}$ a background image. Second, regions occupied by bubbles have to be localized since they must not be considered in concentration measurements. For that, a mask of the bubbles is built by applying a threshold to the spatial gradient of image $I_{c}$. After application of the mask, the gray level of each pixel of $I_{c}$ is converted into a concentration value by the use of Eq. (1). Note that the prefactor $G L(C=0)$ in Eq. (1) is determined from the gray level 


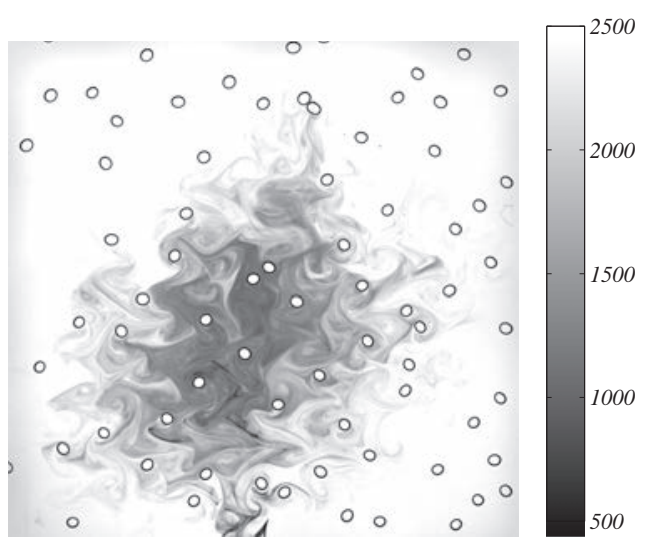

(a) Raw image (Greylevel)

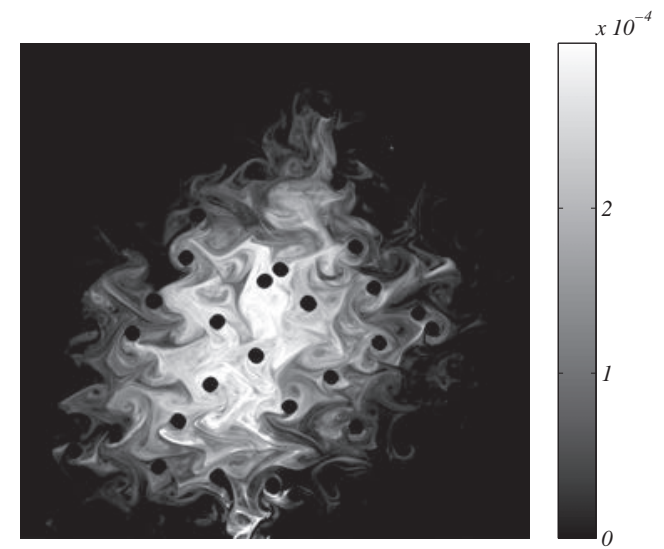

(b) Concentration field $(\mathrm{mol} / \mathrm{L})$

FIG. 3. Illustration of the image processing. Images taken $3 \mathrm{~s}$ after the dye injection beginning for $\alpha=5.3 \%$. Note that lighter means greater concentration in the treated image (to the left), while it is the opposite in the raw image (to the right).

of each pixel before dye injection. An instantaneous field of dye concentration obtained from the raw image is presented in Fig. 3. Using the calibration curve (1) the raw image is transformed into a quantitative measurement of the concentration averaged over the gap.

\section{EXPERIMENTAL RESULTS}

An instantaneous distribution of concentration in the bubble swarm at $\alpha=5.3 \%$ is reported in Fig. 4(a) where black masks are superposed on the bubbles. A few seconds after the injection of the dye, we can notice an important spreading around the central part of the initial concentration field. The topology of the concentration field shares common characteristics with the agitation in the liquid phase described in Bouche et al. [5,8] due to the dominant role of the wakes in the agitation. It is visible in this figure that the concentration field is stretched and distorted due to the passages of the bubbles and that its topology is mainly the signature of the stirring and entrainment by the bubble wakes. In order to achieve a quantitative description of the concentration transport in the bubble swarm, the temporal evolution of two different quantities is studied: the horizontally averaged concentration and the characteristic mixing time.

First, we consider the concentration averaged in the horizontal direction that is plotted in Fig. 4(b) as a function of the vertical direction at three different instants for a single mixing experiment. Note that in order to be consistent with the numerical part, which will be presented later, the concentration is averaged in horizontal the direction over the distance $L$, which is delimited by the two vertical (green) lines in Fig. 4(a). From the spatio-temporal evolution of the concentration field, it is clearly visible in Fig. 4(b) that the maximum concentration is transported upward while the dye spreads around this maximum as time increases. Since the field of view is not large enough to capture the entire concentration spatial distribution, the evolution of its moments (average, variance, skewness) over a long time cannot be directly determined from the experiments. Such a quantitative description will, however, be achieved by means of the model that will be presented in Sec. V.

Second, we consider the mixing time $T_{m}$, which was introduced by Bouche et al. [8] and Alméras et al. [9]. It is calculated from the temporal evolution of the concentration averaged over a fixed delimited area. In this work, we consider a single domain of $40 \times 153 \mathrm{~mm}^{2}$ size, its bottom border being located at $55 \mathrm{~mm}$ from the gas injectors. It is limited by the horizontal (red) lines in Fig. 4(a). It is large enough so that there is no concentration flux that leaves the domain by its lateral borders, and thus only the vertical transport is considered. Figure 4(c) shows the temporal evolution of the concentration 


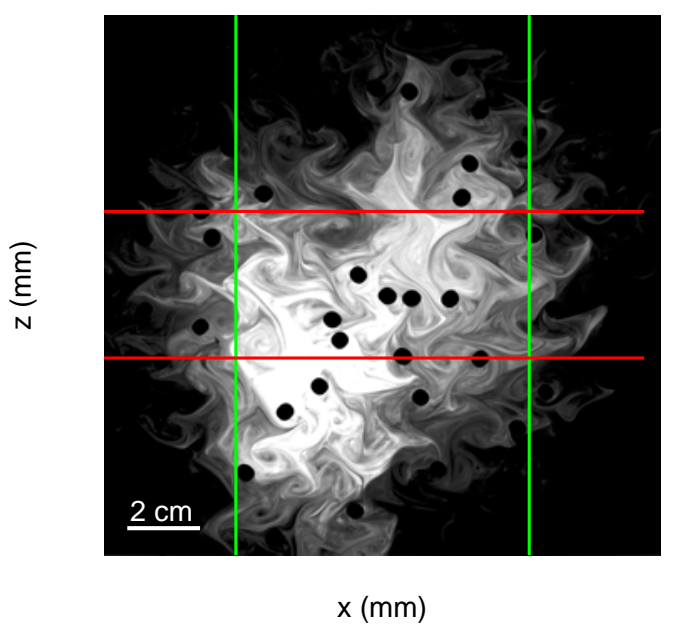

(a)

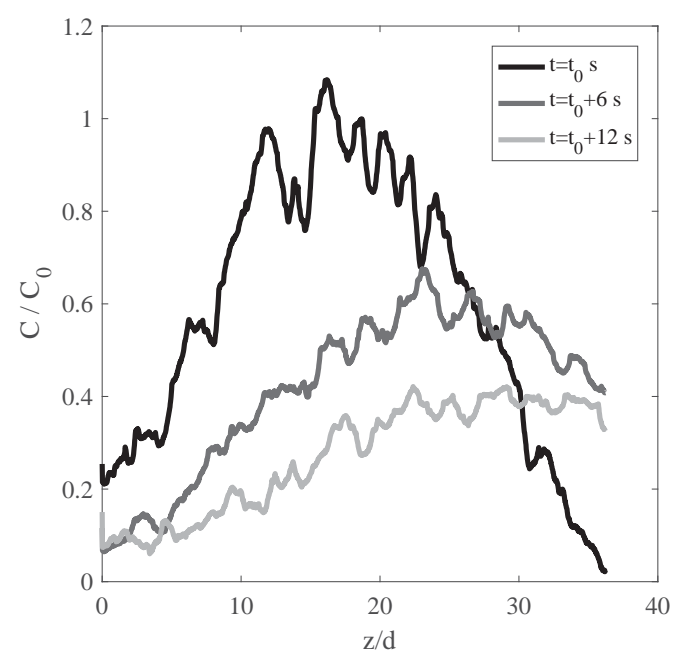

(b)

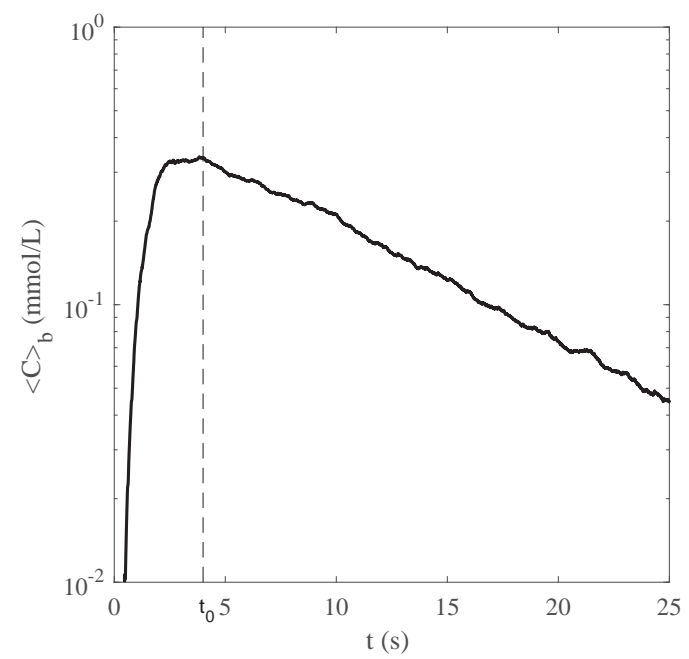

(c)

FIG. 4. Spatial and temporal experimental evolutions of the concentration $(\alpha=5.3 \%)$. (a) Instantaneous concentration field, (b) Vertical concentration profiles at three different instant, and (c) Temporal evolution of the concentration averaged over the region between the two horizontal (red) lines.

$\langle C\rangle_{b}$ averaged over this domain, for a single mixing experiment at a gas volume fraction $5.3 \%$. An exponential decrease of $\langle C\rangle_{b}$ is observed in Fig. 4(c), which has been shown to be incompatible with a mechanism of regular diffusion (Bouche et al. [8] and Alméras et al. [9]). For every experimental run, a mixing time $T_{m i}$ is calculated such as $\langle C\rangle_{b}(t)=\langle C\rangle_{b}\left(t_{0}\right) \times \exp \left[\left(t-t_{0}\right) / T_{m i}\right]$, where $t_{0}$ is the time at which we start investigating the mixing. For each gas volume fraction, the mean mixing time $T_{m}$ is calculated by averaging the times $T_{m i}$ over five independent runs. Figure $7(\mathrm{~b})$ represents the average mixing time $T_{m}$ as a function of the gas volume fraction where the error bars are estimated from the standard deviation of the five similar runs. We see that the mixing time decreases with increasing gas volume fraction as $T_{m}=\tau_{m} / \alpha$ (with $\tau_{m}=55 \mathrm{~s}$ in the present work), which is in agreement with previous measurements.

In order to develop a model able to reproduce these spatial and temporal evolutions of the concentration field, the elementary mechanisms responsible for the mixing in a confined bubbly flow 


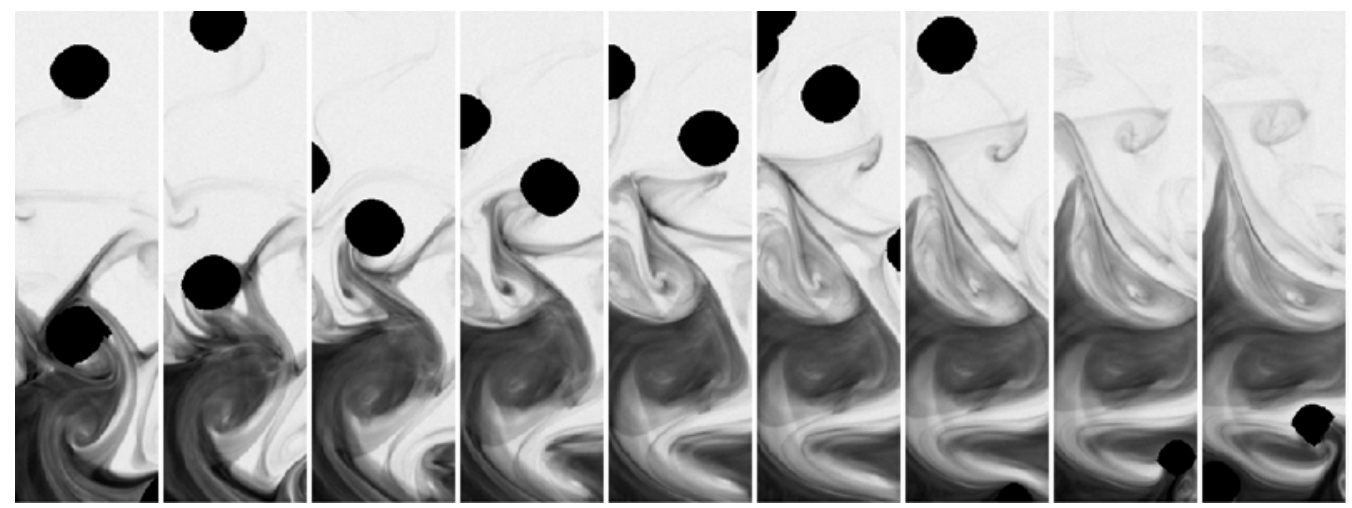

FIG. 5. Illustration of the mixing process by capture of the dye in the bubble wakes. The time step between two images is $0.04 \mathrm{~s}$.

have to be identified and characterized. The similarity between the distribution of the concentration and the liquid velocity field noted by Bouche et al. [8] was a first indication of the important role of the localized wakes on the dye transport. In addition, time-resolved measurements of the concentration $C(t)$ at a given point obtained by LIF by Alméras et al. [9] showed that concentration fluctuations share common statistical properties with velocity fluctuations: asymmetric PDFs and spectrum showing a $k^{-3}$ slope (where $k$ is the wave number), which can both be explained by wakes of randomly distributed bubbles crossing intermittently the point of measurement (Risso [16,17]). A careful examination of a sequence of images taken in the present experiments reveals the elementary steps occurring during dye transport. Figure 5 shows a sequence of nine images (separated by an interval of $0.04 \mathrm{~s}$ ) taken when a bubble is passing from a region of high dye concentration layer (in black) to a region of low concentration (in white). It is seen that the dye distribution is mostly entrained at the bubble rear where it is then deformed, stretched, and folded. Two stages can be distinguished. During the first stage, which corresponds to the first four images in the Fig. 5, the dye is mainly entrained in the bubble wake while a moderate deformation of the background concentration field around the bubble at distances of about one diameter is also observed. The second stage, which corresponds to the last four images, starts when the bubble travels over a few diameters from the dye layer. The liquid motion vanishes under the action of the wall friction, and the dye pattern progressively freezes and no longer follows the bubble. Mixing in a thin-gap cell therefore mainly results from the direct interaction of the dye with the flow in the close vicinity of the bubbles, the main effect being that it is transported for a finite time in the vertical direction. It is thus an intermittent and convective process for which we propose to model it by considering that each bubble entraps a volume of dye in its wake, transports it over a certain length $L$, and finally releases it.

\section{NUMERICAL MODEL}

A one-dimensional intermittent numerical model of transport has been developed to describe the global evolution of the concentration distribution $C(z, t)$ in the vertical direction due to the mixing by the capture of dye in bubble wakes. The present model has been designed to describe the mixing at a mesoscale, which is smaller than that of the global evolution of the concentration distribution but larger than that of the concentration fluctuations resulting from the passage of each individual bubble (Alméras et al. [9]). This leads us to consider a one-dimensional regular grid of mesh size $L^{2}$ (Fig. 6). Obviously, this model requires us to introduce a subgrid model in order to describe the effect of scales lower than $L$. This subgrid model is based on the mixing mechanism described in Sec. III. It assumes that each bubble transports, over a length $L$, without external exchange, a given volume of liquid $A_{t} \times w$ within its wake, while it rises at a velocity $V$. Assuming that each bubble 


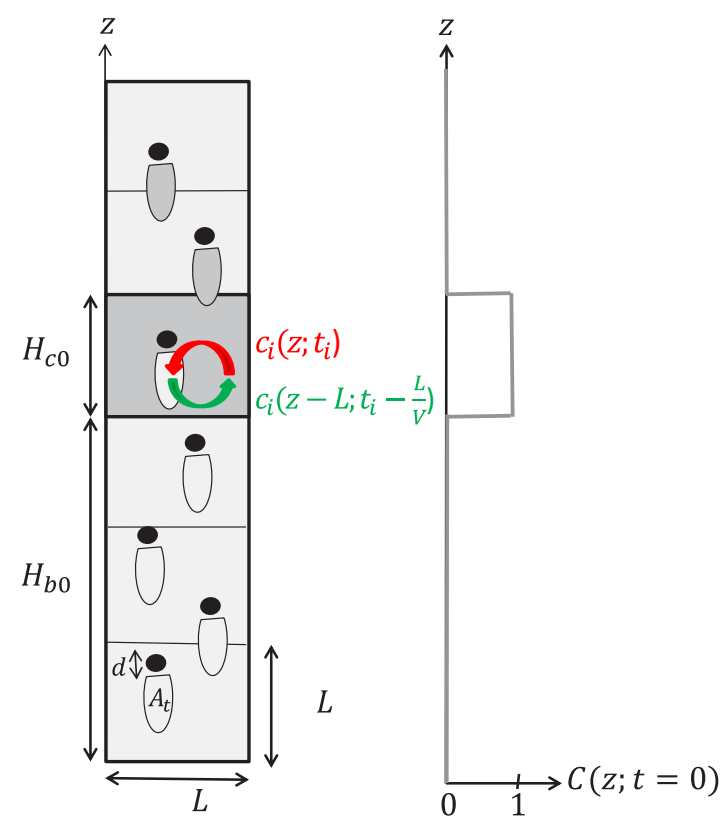

FIG. 6. Illustration of the numerical model. The initial concentration profile is indicated to the right.

transports dye patches independently, the summation over a large number of bubbles is carried out in order to simulate the mixing by a bubble swarm. Consequently, the model involves four physical parameters, which are the rising velocity $V$ of the dye patches, the number of dye patches per unit area $N^{*}$, the transported volume $A_{t} \times w$, and the length of transport $L$. It is important to note that, while $V$ and $N^{*}$ are directly measured in experiments, $A_{t}$ and $L$ are not. They will be estimated later (Sec. VI) from comparison between the experimental and simulated evolutions of the distribution of the concentration.

The simulation is initialized as follows. The initial concentration field $C(z ; t=0)$ corresponds to a concentration $C_{0}$ within a layer of thickness $H_{c 0}$, which corresponds to the region where the dye has been injected. Below and above this region the concentration is equal to zero (see Fig. 6 to the right). The bubbles are initially inserted in the region of height $H_{b 0}$ located below the region containing the dye. $H_{b 0}$ is chosen large enough so that there are still bubbles below the initial dye region at the end of a simulation. This allows us to carry on with transport all along the numerical simulation. The number of bubbles, $N=N^{*} L H_{b 0}$, is determined from the number of dye patches per units of area $N^{*}$. Their positions $z_{i}$ are randomly chosen independently from each other so as to obtain a statistically homogeneous distribution. The concentration in the volume of liquid transported by each bubble is taken equal to the concentration in the liquid at the bubble location, $c_{i}(t=0)=C\left(z_{i} ; t=0\right)$. Moreover, an initial time delay $t_{i}$ is attributed to each bubble, which corresponds to the time elapsed from the instant of its last dye capture. These times are randomly chosen such as to be uniformly distributed between 0 and $L / V$.

The simulation advancement is done by steps of $\delta t=L /\left(V N_{t}\right)$, where $N_{t}$ is an integer which defines the time resolution. The influence of $N_{t}$ has been tested, and it turns out that the results become independent of $N_{t}$ provided it is larger or equal to 60. At each time iteration $k$, two numerical operations are performed successively. First, each bubble $i$ is moved upward by $V \Delta t$, as $z_{i}(t+\delta t)=z_{i}(t)+V \Delta t$. Second, it is checked whether it is time for this bubble to capture a fresh volume of liquid. For that purpose, a counter $\phi_{i}$ is introduced and defined as $\phi_{i}=\left(t_{i}+k \times \delta t\right) V / L$. If the integer part of $\phi_{i}$ is the same as that of the previous time step, the concentration of liquid transported by the bubble $i$ is unchanged: $c_{i}(k \times \delta t)=c_{i}[(k-1) \times \delta t]$. If the integer part of $\phi_{i}$ 


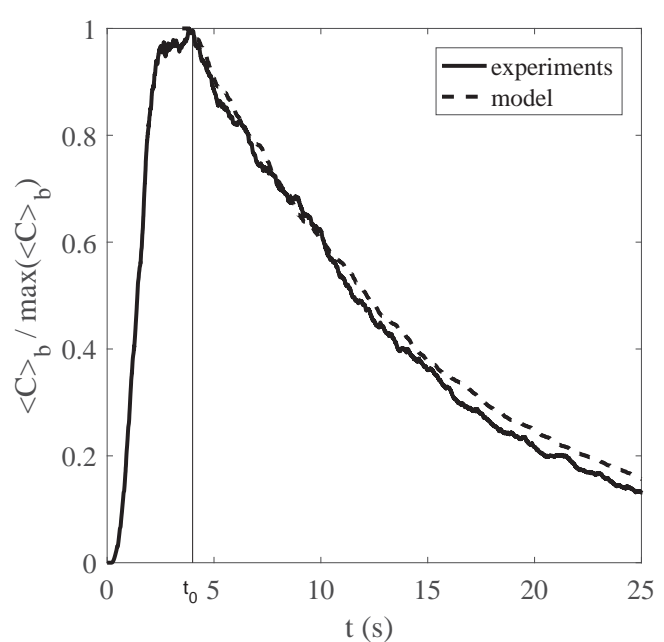

(a)

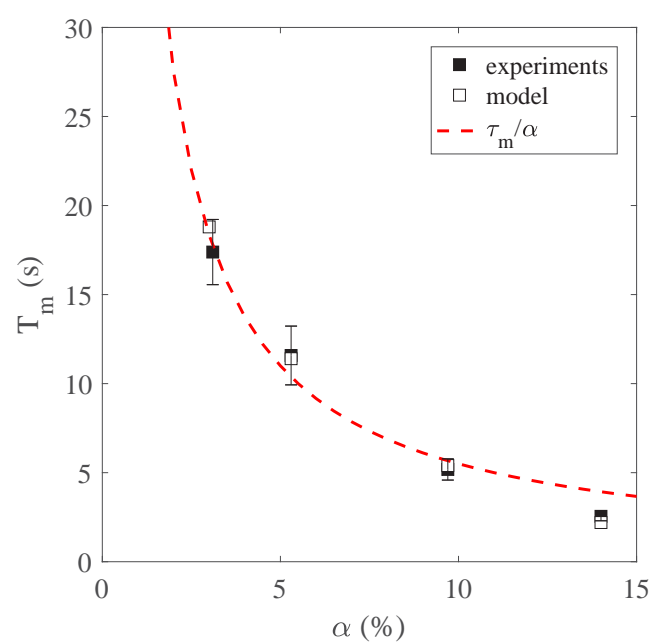

(b)

FIG. 7. Model validation from the global mixing time $T_{m}$. (The model parameters are given in Table I.) (a) Temporal evolution of the concentration averaged over a fixed area. $\alpha=5.3 \%$ and (b) Evolution of the mixing time $T_{m}$ with $\alpha$.

has been incremented, the bubble releases the transported liquid and captures a new one at the concentration of the cell where it is located: $c_{i}(k \times \delta t)=C[z=k \delta t V ; t=(k-1) \times \delta t]$. When the time advancement has been done for all the bubbles, a new concentration field $C(z=k \delta t V ; t=k \delta t)$ is calculated by accounting for the exchanges with all the bubbles in each cell.

\section{COMPARISON BETWEEN THE MODEL AND THE EXPERIMENTS}

The mixing model requires the knowledge of both the length of transport $L$ and the transported volume $A_{t} \times w$. As mentioned in Sec. IV, these two parameters are not directly obtained from the experiments. They have to be determined by a comparison between the model prediction and the experimental results. This comparison not only aims at a validation of the model, but also aims at establishing the model and its parameters on robust bases so that it can be used to learn more about the physics of mixing through numerical simulations (see Sec. VII). The comparison of the model with the experiments is based on the analysis of the two quantities presented in Sec. III: the mixing time and the spatio-temporal evolution of the concentration distribution in the vertical direction. But, before comparing the model and the experiments, some adjustments have to be performed for both the experiments and the model in order to ensure that the same quantities are measured.

It has been observed by Alméras et al. [9] that the mixing time $T_{m}$ depends widely on the vertical position of the measurement volume. The further the location of the measurement volume from the tip injector is, the larger the mixing time is (see Fig. 10 in Alméras et al. [9]). It is thus important to locate the area where the mixing time is calculated at the same height for both the experimental and the numerical approaches. In the present work, the domain of interest has been located just above the top border of the dye layer after the dye injection. This corresponds approximately to $55 \mathrm{~mm}$ above the gas injector tip in the experiments. Once the area of interest is located, a spatial average of the concentration field, depending on time, is performed over a fixed domain. It has been shown that the size and the shape of the domain is not a significant parameter for the calculation of the mixing time (Alméras [18]). For ease of calculation, we calculate the averaged concentration over a domain size of $L \times L$ for the numerical simulations and of $40 \times 153 \mathrm{~mm}^{2}$ for the experiments [Figs. 4(a) and 4(c)]. A first result is that the model predicts an exponential decrease of the concentration averaged over a finite region [Fig. 7(a)] and can accurately match the experimental result for a well chosen 


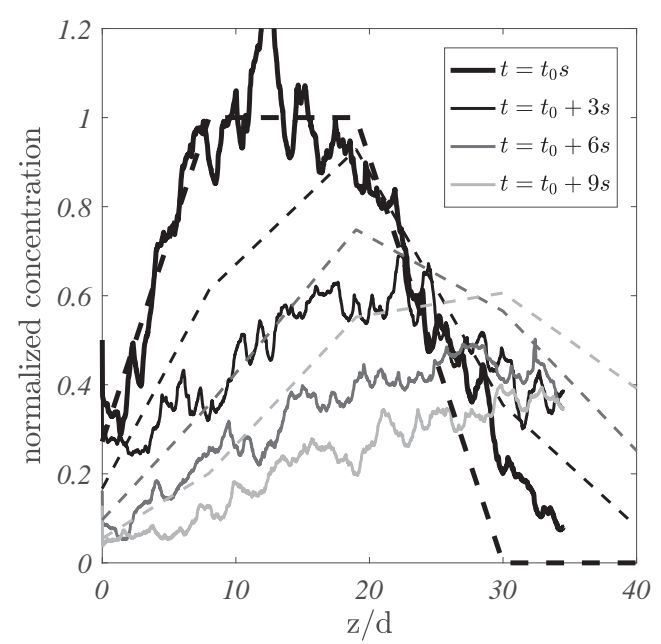

(a) $A_{t}=0.8 A_{b}, L=11 d$

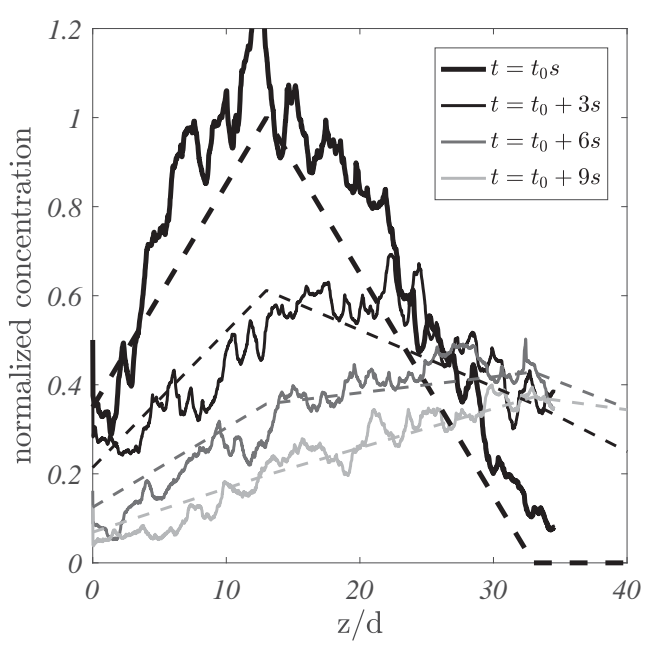

(b) $A_{t}=2 A_{b}, L=23 d$

FIG. 8. Influence of the parameter pair $\left(A_{t} ; L\right)$ on the concentration profiles for $\alpha=9.7 \%$. While the two pairs allow the model to predict the experimental global mixing time (equal to $5.4 \mathrm{~s}$ for the present gas volume fraction), only one allows it to reproduce the concentration profiles.

set of parameters. A mixing time can thus be estimated from both the experimental and numerical concentration fields.

A one-dimensional vertical profile of the concentration has to be defined from the experimental concentration field $C(x ; z ; t)$ in order to be comparable with the one-dimensional concentration profile calculated by the model, which is integrated over a length $L$. It is important to stress that since the mixing experiments are reproducible (Fig. 7), we could base our spatio-temporal analysis on a single mixing experimental run. The one-dimensional experimental profile $C(z ; t)$ is therefore calculated as follows:

$$
C(z ; t)=\int_{-L / 2}^{L / 2} C(x ; z ; t) d x .
$$

The model predictions obviously depends on the pair $\left(L ; A_{t}\right)$, which is a function of the gas volume fraction but is independent of time. This suggests using the global mixing time $T_{m}$ and the spatiotemporal evolution of the concentration distribution to determine the model parameters. For that purpose, the following two-step method has been developed. First, the global mixing time $T_{m}$ is used to fit the model parameters. This turns out to be insufficient because, for a given gas volume fraction, the experimental characteristic time $T_{m}$ can be obtained for a several pairs $(L ; A t)$. As an example, for $\alpha=9.7 \%$, both pairs $\left(A_{t} ; L\right)=\left(0.8 A_{b} ; 11 d\right)$ and $\left(A_{t} ; L\right)=\left(2 A_{b} ; 20 d\right)$ allow us to reproduce the experimental global mixing time $T_{m}$, which is equal to $5.4 \mathrm{~s}$. This step allows us nevertheless to define a restricted ensemble of pairs $(L ; A t)$. In a second time, a unique pair is selected from this restricted ensemble by minimizing by means of the least-square method the difference between the numerical and experimental concentration profiles considered at various times. Eventually, it turns out that only a single pair $(L ; A t)$ is able to reproduce both the propagation and the spreading of the one-dimensional experimental profile of concentration over time. Figure 8 clearly shows that if the model matches well the experimental concentration profiles for $\left(A_{t} ; L\right)=\left(2 A_{b} ; 23 d\right)$, it fails for $\left(A_{t} ; L\right)=\left(0.8 A_{b} ; 11 d\right)$.

Finally, for each investigated gas volume fraction, a unique pair of values of $L$ and $A t$ has been determined. The corresponding pairs are presented in Table I. With these values, Fig. 7 shows a good agreement between simulated and measured values of $T_{m}$, the small discrepancies being 
TABLE I. Transported area $\left(A_{t}=V_{t} / w\right)$ and transport length $(L)$ for various gas volume fractions $\alpha$.

\begin{tabular}{lcccc}
\hline \hline$\alpha$ & $3 \%$ & $5.3 \%$ & $9.7 \%$ & $14 \%$ \\
\hline$L / d$ & $25 \pm 2$ & $23 \pm 2$ & $20 \pm 2$ & $14 \pm 1$ \\
$A_{t} / A_{b}$ & $2 \pm 0.5$ & $2 \pm 0.5$ & $2 \pm 0.5$ & $2 \pm 0.5$ \\
\hline \hline
\end{tabular}

mainly due to the discrete scan of $(L ; A t)$ pairs. A satisfactory agrement between the model and the experiments is also observed in Fig. 9 where the experimental concentration profiles are compared to the numerical predictions at different instants. The concentration profiles are normalized by the maximum concentration at initial time. Note that the initial concentration profile for the simulation has been designed so that it matches the experimental concentration profile at the end of the dye injection. The agreement between the model and the prediction is satisfactory even at large gas volume fractions where the concentration in the window of observation is very dilute, the maximum barely reaching $10 \%-15 \%$ at $t=11 \mathrm{~s}$. The main discrepancies are mainly due to the size of the numerical mesh, which increases as the gas volume decreases. We can therefore be confident that the model reproduces well the mixing properties in a Hele-Shaw cell, even at larger times.

\section{PHYSICAL INTERPRETATION OF THE MODEL PARAMETERS}

The comparison between the model and the experiments allows us to determine the two unknown parameters of the model, the transported volume $A_{t} \times w$ and the length of transport $L$, for the four investigated gas volume fractions ranging from $3.0 \%$ to $14.0 \%$ (Table I). In the present section, we will discuss the evolution of these two parameters with the gas volume fraction and relate them to physical quantities describing the liquid flow.

The length of transport shows two distinct regimes (Table I). For low gas volume fractions $(\alpha \leqslant$ $5 \%$ ), it is almost constant and close to the viscous length $l_{v}=V w^{2} / v \approx 25 d$ introduced in previous works (Roig et al. [14], Bouche et al. [5]). This viscous length corresponds to the time required for a vortex rising initially at velocity $V$ to be damped by the shear induced by the walls. For a larger gas volume fraction $(\alpha \geqslant 9 \%)$, the length of transport no longer scales with the viscous length and decreases with $\alpha$. This decrease will be related in Sec. VII to the time between successive bubble passages.

Regarding the transported volume, it turns out to be independent of the gas volume fraction and equal to twice the bubble volume $\left(A_{t}=2 A_{b}\right)$. This value roughly corresponds to the dimension of the average wake of a bubble immersed in a confined bubble swarm measured in previous works (see Fig. 3 of Bouche et al. [5]).

\section{MIXING DESCRIPTION FROM THE MOMENTS OF THE CONCENTRATION DISTRIBUTION}

The natural way of describing the transport and mixing of a chemical component in a flow consists of using an advection-diffusion equation. This requires one first to assess whether or not the mixing can be described by such an equation and, second, to determine the propagation velocity and the effective diffusivity. These two points can be addressed by characterizing the temporal evolution of the centered moments of the spatial distribution of the concentration. In fact, the first-order moment describes the overall motion, the second-order moment the spreading, and the third-order moment the asymmetry of the distribution. In the following, these three moments are calculated only from the numerical model. Indeed, they cannot be calculated from the experimental profiles, which are available for too limited spatial and time ranges. It is clear from Fig. 9 that the size of the measurement window is not enough large to compute the statistical moments of the distribution. Moreover, because of the existence of a average transport in the upward direction, the dye is present in the measuring window during only $30 \mathrm{~s}$. On the other hand, the model provides nontruncated concentration profiles over a time period larger than $100 \mathrm{~s}$. For those reasons, we now use the results 


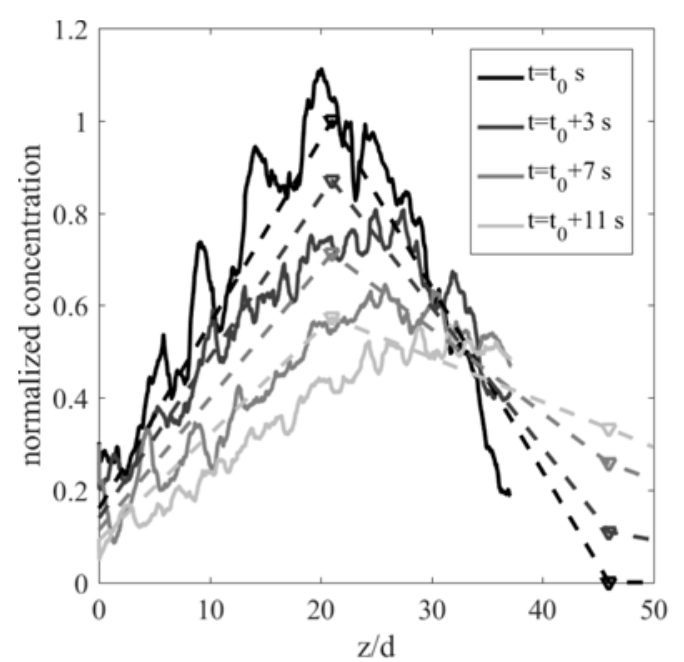

(a) $\alpha=3.0 \%$

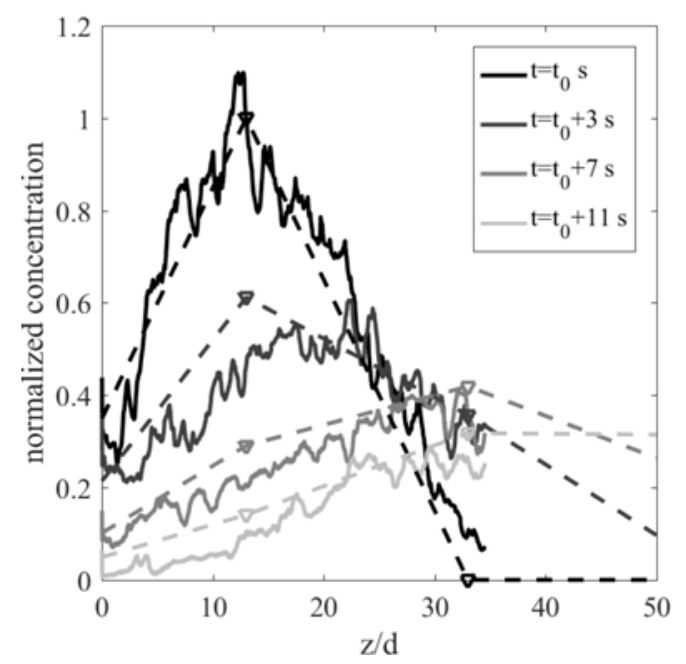

(c) $\alpha=9.7 \%$

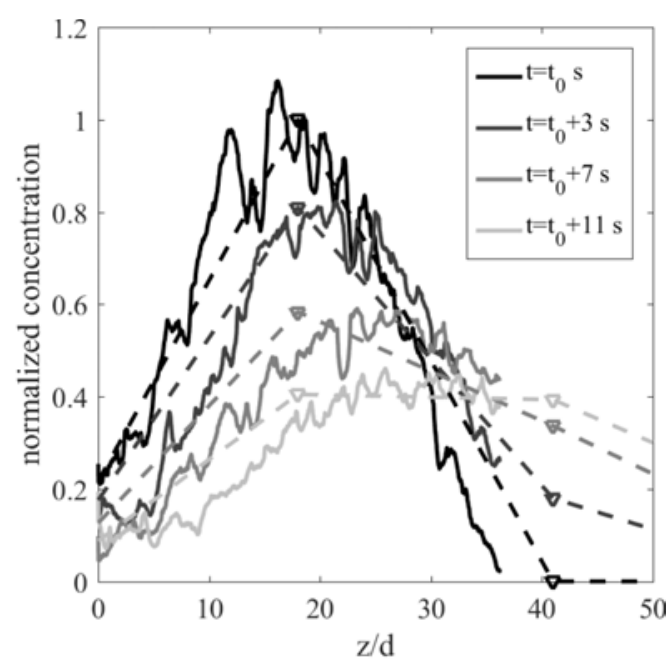

(b) $\alpha=5.3 \%$

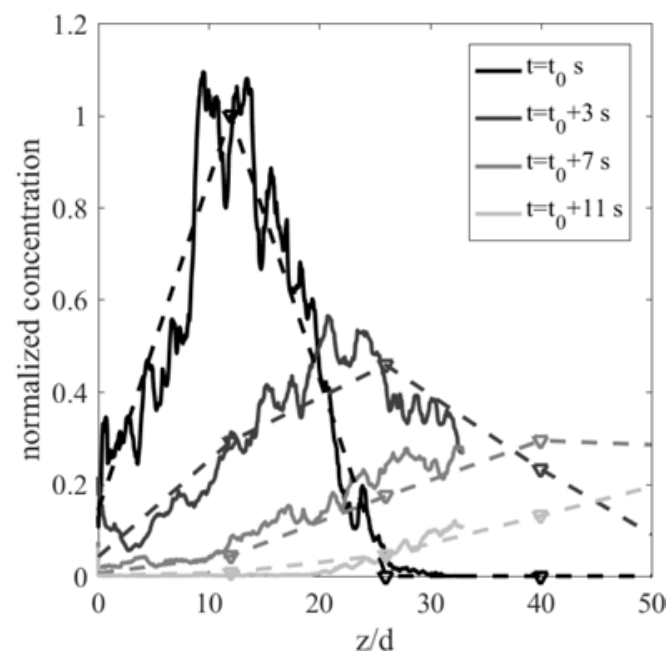

(d) $\alpha=14.0 \%$

FIG. 9. Model validation from one-dimensional concentration profiles at different instants. Continuous lines: experiments. Dashed lines: model.

of the model obtained with the parameters adjusted on the experiments for each gas volume fraction to enhance the discussion of the mixing induced by a confined bubbly flow.

\section{A. Overall motion of the concentration distribution}

The overall motion is given by the movement of the position of the centered first-order moment $z_{b}(t)$ of the concentration distribution $C(z ; t)$, usually called center of mass:

$$
z_{b}(t)=\frac{\int_{-\infty}^{+\infty} z C(z ; t) d z}{\int_{-\infty}^{+\infty} C(z ; t) d z} .
$$

The temporal evolution of the center of mass is plotted in Fig. 10(a) and shows a linear evolution for all gas volume fraction. A mean propagation velocity, $V_{p}=d z_{b} / d t$ is thus estimated for the four studied gas volume fractions. Once normalized by the bubble velocity $V$, the propagation velocity 


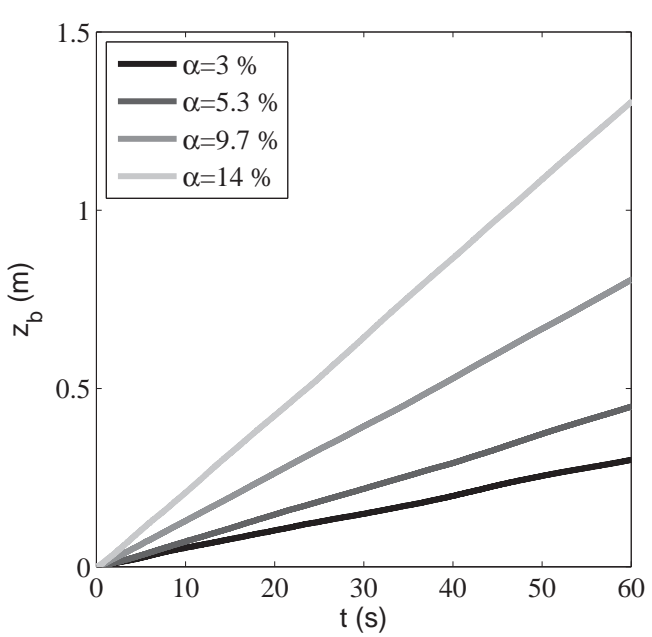

(a)

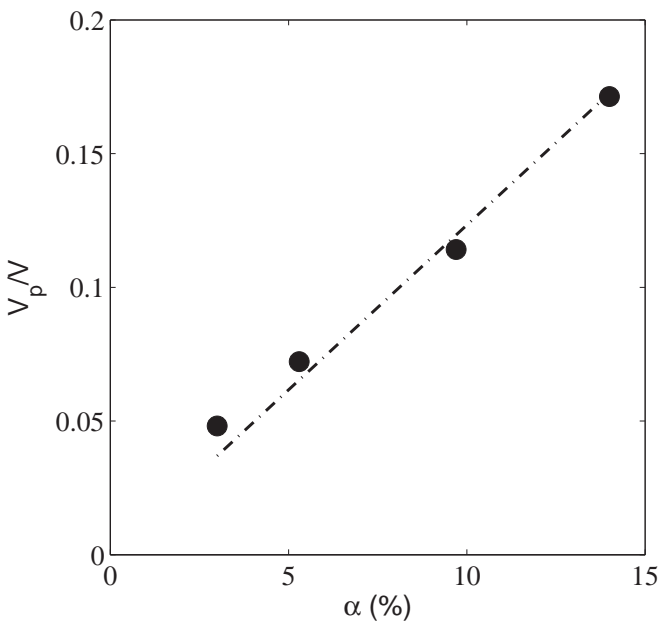

(b)

FIG. 10. Global displacement of the vertical distribution of the concentration. (a) Temporal evolution of the barycentre of the concentration distribution and (b) Propagation velocity normalized by the mean bubble velocity.

is observed to increase linearly with the gas volume fraction [Fig. 10(b)]:

$$
V_{p}=k_{p} V \alpha,
$$

where $k_{p}$ is found equal to 1.1 .

The physical origin of this propagation velocity still remains unclear. The sum of liquid volumes entrained in the bubble wakes corresponds to a total upward liquid flow rate $Q_{l}=V_{t} / V_{b} \alpha V w W$, where $W$ is the width of the cell. Since the liquid flow rate in every horizontal section of the cell is zero, this upward flow has to be compensated by an equivalent downward flow. This downward flow can either be located in the regions between the bubbles or localized in two thin regions close to the cell lateral borders. In the second case, there should exist an upward mean liquid velocity in the center of the cell $V_{l}=V_{t} / V_{b} \alpha V=2 \alpha V$, which is approximately twice the propagation velocity of the concentration profile. Unfortunately, the present results do not allow to conclude whether the propagation velocity $V_{p}$ is partly or completely due to the existence of a mean liquid velocity far from the cell walls.

\section{B. Spreading of the concentration distribution}

The spreading of the dye is described by the temporal evolution of the centered second-order moment $\sigma^{2}$ of the concentration distribution:

$$
\sigma^{2}(t)=\frac{\int_{-\infty}^{+\infty}\left[z-z_{b}(t)\right]^{2} C(z) d z}{\int_{-\infty}^{+\infty} C(z ; t) d z} .
$$

Figure 11(a) shows a linear evolution of $\sigma^{2}$ with time, which means that an effective diffusivity can be estimated as $D=\frac{1}{2} \frac{d \sigma^{2}}{d t}$. Figure 11(b) shows the effective diffusivity for the four studied gas volume fractions. We can see that the effective diffusion coefficient, which is of the order of $10^{-4} \mathrm{~m}^{2} / \mathrm{s}$, is much higher than the molecular diffusion coefficient. The mixing by the bubbles is therefore the dominant mechanism.

Figure 11(b) shows the evolution of $D$ with the gas volume fraction. Two regimes are visible. The diffusion coefficient first increases with $\alpha$ at low gas volume fraction before reaching a constant value. 


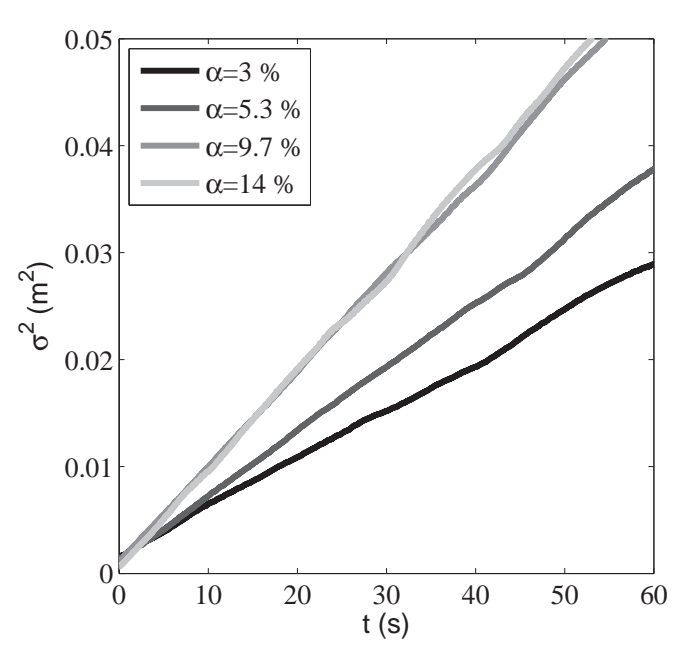

(a)

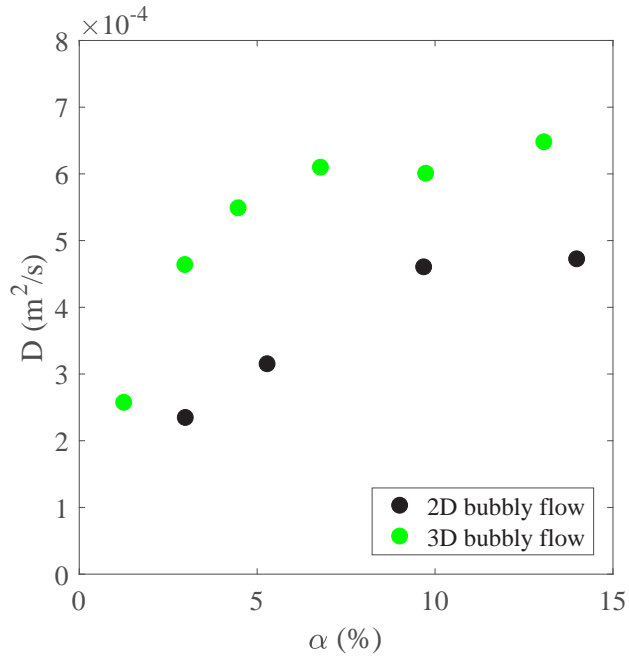

(b)

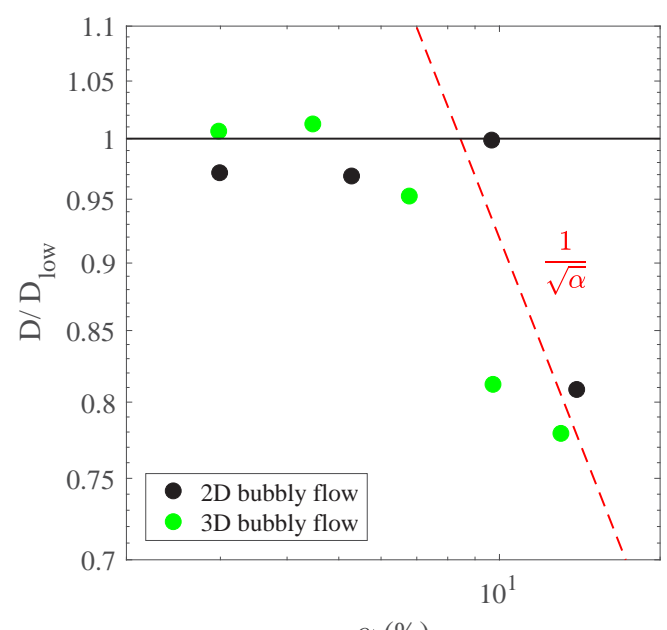

$\alpha(\%)$

(c)

FIG. 11. Spreading of the concentration distribution. (a) Temporal evolution of the variance of the concentration distribution, (b) Diffusion coefficient as a function of the gas volume fraction $\alpha$, and (c) Diffusion coefficient normalized by $D_{\text {low }}$ as a function of the gas volume fraction $\alpha$.

The transition occurs around $\alpha \approx 10 \%$. Figure 11 (b) also reports the vertical diffusion coefficient measured by Alméras et al. [3] in a three-dimensional bubble column, where the bubbles diameter ranges from 2.1 to $3.9 \mathrm{~mm}$, and the Reynolds number is approximately 750 . It is also worth mentioning that in recent numerical simulations of the mixing induced by a tridimensional bubble swarm at $\alpha=2.4 \%$ and $\operatorname{Re}=30$ by Loisy et al. [19] a diffusion coefficient was also measured in the vertical direction of the same order of magnitude as the present results at equivalent Péclet number. Since the parameters of the two- and three-dimensional configurations are not exactly the same, a direct quantitative comparison of the values of $D$ is not relevant. However, it is remarkable that they are of the same order of magnitude and show a similar evolution with $\alpha$. This suggests modeling the diffusion coefficient in a similar way as Alméras et al. [3]; as the product of the standard deviation 
$u^{\prime}$ of the velocity fluctuations in the liquid phase by their characteristic length of correlation $\Lambda$ :

$$
D \propto u^{\prime} \Lambda \text {. }
$$

At low gas volume fraction, it is reasonable to think that $\Lambda$ scales as the length of the wakes, which corresponds to both the viscous length $l_{v}$ and the transport length of dye patches (see Sec. VI). The diffusion coefficient is thus expressed as

$$
D_{\text {low }}=k_{\text {low }} u^{\prime} \Lambda_{\text {low }}
$$

where $\Lambda_{\text {low }}=l_{v}$. The prefactor $k_{\text {low }}$ is a dimensionless constant, and the standard deviation of the liquid velocity fluctuations has been measured by Bouche et al. [5]: $u^{\prime}=1.07 V_{s b} \alpha^{0.46}$, where $V_{s b}$ is the terminal rising velocity of an isolated bubble. Figure 11(c) shows the present diffusion coefficient $D$ normalised by the value of $D_{\text {low }}$ given by Eq. (8) with $k_{\text {low }}=0.01$. The good matching of $D$ and $D_{\text {low }}$ up to $\alpha \approx 10 \%$ allows us to conclude that it is relevant to consider a constant length scale $\Lambda$ and that the increase of $D$ is due to only the increase of the velocity fluctuations. In a three-dimensional bubble column, it has been shown that at $\alpha \approx 6 \%$, the correlation length is limited by the average time $\Delta T$ between the passage of two consecutive bubble leading to express $\Lambda$ as $\Lambda=u^{\prime} \Delta T$. At large volume fraction, the diffusion coefficient could thus be written as

$$
D_{\text {high }}=k_{t} u_{i}^{2 \prime} \Delta T \text {. }
$$

Since $\Delta T$ scales as $\alpha^{-1}$ and $u^{\prime}$ approximately as $\alpha^{-0.5}, D$ is expected to be approximately independent of $\alpha$ and $D / D_{\text {low }}$ to decrease as $\alpha^{-0.5}$. This is in agreement with the evolution of $D$ and $D / D_{\text {low }}$ observed in Figs. 11(b) and 11(c) at large gas volume fraction. Figure 11(c) also shows that, when properly normalized, the diffusion coefficient measured in a two-dimensional bubble column overlaps remarkably the one measured in a three-dimensional bubble column, even if the underlying mixing mechanisms strongly differ from a configuration to the other. Both coefficients can be modeled by the expressions (8) at low $\alpha$ and (9) at large $\alpha$, provided that the values of $\Lambda_{\text {low }}$ and $u^{\prime}(\alpha)$ of the corresponding configuration are considered.

Now we compare the present results with previous studies that have considered a Hele-Shaw cell. Eames and Bush [6,7] modeled the diffusion coefficient $D_{p}$ of the mixing induced by the drift volume generated by the potential flow around a compound made by the bubble and its stable closed wake as $D_{p}=C_{m}^{\prime}(1+\epsilon) L_{p} V \alpha$, where $C_{m}^{\prime}$ is the added mass coefficient of the compound body, $(1+\epsilon) \alpha$ is the volume fraction of the compound, and $L_{p}$ is a characteristic length of the longitudinal displacement. Assuming $C_{m}^{\prime} \approx 1,1+\epsilon \approx 2$ and $L_{p}=d / 2$, this leads to $D_{p} \approx d V \alpha$, which is in good agreement with the experiments carried out by these authors for bubbles with a stable close wake but predicts a value greater than seven times smaller than that found here. The capture and release of the dye by unstable open wakes therefore generate a much more efficient mixing than the potential flow around the compound made by a bubble and its close stable wake.

\section{Asymmetry of the concentration distribution}

The symmetry of the concentration distribution is analyzed by using the skewness Sk, which is the normalized centered third-order moment:

$$
\operatorname{Sk}(t)=\frac{1}{\sigma(t)^{3}} \frac{\int_{-\infty}^{+\infty}\left[z-z_{b}(t)\right]^{3} C(z ; t) d z}{\int_{-\infty}^{+\infty} C(z ; t) d z} .
$$

Figure 12 shows the temporal evolution of the skewness for each gas volume fraction. As long as the simulation is running $(t \leqslant 100 \mathrm{~s})$, the skewness value remains significant, except at the beginning of the simulation since a symmetric concentration profile is used as initial condition. The concentration distribution is therefore highly asymmetric in the vertical direction, meaning that mixing is incompatible with a purely diffusive process during the simulation time. We can nevertheless observe that the two first moments of the concentration distribution evolve both linearly 


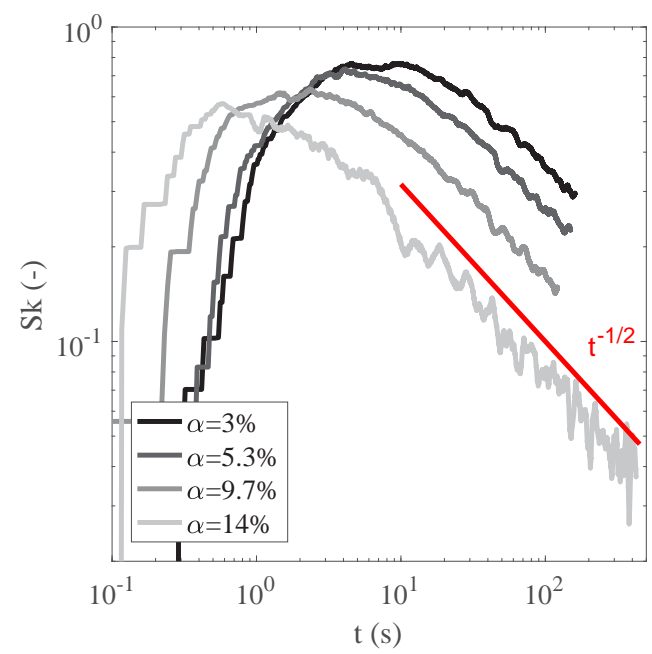

FIG. 12. Skewness of the concentration distribution as a function of time for different $\alpha$.

with the time $t$ and that the skewness tends to decrease as $t^{-1 / 2}$. This means that the concentration distribution will finally tend towards a normal distribution and mixing would evolve towards a diffusive mechanism. However, this diffusive stage is achieved at a very long time compared to the duration of the mixing experiments, which is around $40 \mathrm{~s}$. In fact, considering the time for which $S_{k}=5 \%$, we find that the diffusive regime is reached at $250 \mathrm{~s}$ after the injection for $\alpha=14 \%$, which is the gas volume fraction for which the convergence towards the diffusive stage is obviously the fastest. Considering reasonable timescale regarding the mixing experiments, the present mixing mechanisms therefore involve not only diffusion but also dispersion.

The existence of this asymmetry is a major difference with the mixing in the three-dimensional bubble column, where no asymmetry could be detected even at times smaller than $1 \mathrm{~s}$. The presence of turbulence in an unconfined configuration enhances the random character of the flow and probably accelerates the convergence towards a Gaussian distribution and a regular diffusive process.

\section{CONCLUSION}

A large-scale one-dimensional model based on an intermittent convective transport has been developed to predict the mixing of a passive scalar in a confined bubbly flow. This model assumes that each bubble entraps a volume $V_{t}$ of dye in its wake and transports it along a length $L$ before releasing it. By comparing the model to experiments, these two parameters have been estimated. The volume $V_{t}$ is found to be constant and equal to twice the bubble volume, which corresponds nearly to the size of the region of velocity deficit at the rear of a bubble (Bouche et al. [5]). The length of transport $L$ is related to the correlation length of the dye fluctuations and shows two regimes. For $\alpha \leqslant 5 \%, L$ is close to the viscous length $l_{v}=V w^{2} / \nu$, whereas it is limited by the successive bubble passages at larger gas volume fractions.

Mixing experiments have been performed in a Hele-Shaw cell by measuring the absorption of the light emitted by a LED panel. Spatial and temporal evolutions of the concentration distribution in the vertical direction have thus been determined experimentally. They show an upward propagation and a vertical spreading, which have been well reproduced by the model. The model has then been used to characterize the properties of the mixing process from the analysis of the centered moments of the concentration distribution. An upward propagation velocity, which is small compared to the bubble velocity and scales as $V \alpha$, has been found. The spreading of the concentration distribution has been characterized by a diffusion coefficient which is of this order of $5 \times 10^{-4} \mathrm{~m}^{2} / \mathrm{s}$ and depends upon the 
gas volume fraction. Similarly to the case of a three-dimensional bubble column (Alméras et al. [3]), the effective diffusivity can be written as the product of the standard deviation of the liquid velocity fluctuations by a characteristic correlation length. At low gas volume fraction, the correlation length is the viscous length, which is independent of the gas volume fraction. This leads to an evolution of the effective diffusivity as the square root of the gas volume fraction. On the other hand, at large gas volume fraction, the correlation length is related to the time interval between successive bubbles, in a saturation of the effective diffusivity at high gas volume fraction. This phenomenon of decorrelation of the fluctuations by the bubble disturbances is similar in two- or three-dimensional bubble columns and therefore independent of the precise nature of the fluctuations responsible for the mixing. The model for the effective diffusion coefficient originally proposed by Alméras et al. [3] for a three-dimensional bubble column can thus be generalized to other configurations of bubbly flow provided that the properties of the liquid fluctuations (integral length scale and standard deviation) are known for this specific flow.

Even if an effective diffusivity has been measured and that the concentration distribution would finally tend toward a normal distribution, it is important to stress that the present mixing mechanism in a two-dimensional bubble column is not a purely diffusive process because of the existence of a strong asymmetry in the concentration distribution. A large-scale modeling of such a process by means of a transport equation of the concentration should include a dispersive term (third-order derivative) in addition to the classic diffusive term (second-order derivative).

\section{ACKNOWLEDGMENT}

The authors thank Mickaël Bazin for his contribution to the development of the image processing.

[1] T. Willmarth and M. Ishii, Two-phase flow regimes in narrow rectangular vertical and horizontal channels, Int. J. Heat Mass Transfer 37, 1749 (1994).

[2] P. Spicka, M. M. Dias, and J. C. B. Lopes, Gas-liquid flow in a 2D column: Comparison between experimental data and CFD modelling, Chem. Eng. Sci. 56, 6367 (2001).

[3] E. Alméras, V. Roig, F. Risso, C. Plais, and F. Augier, Mixing by bubble-induced turbulence, J. Fluid Mech. 776, 458 (2015).

[4] E. Alméras, C. Plais, F. Euzenat, F. Risso, V. Roig, and F. Augier, Scalar mixing in bubbly flows: Experimental investigation and diffusivity modelling, Chem. Eng. Sci. 140, 114 (2016).

[5] E. Bouche, V. Roig, F. Risso, and A.-M. Billet, Homogeneous swarm of high-Reynolds-number bubbles rising within a thin gap. Part 2. Liquid dynamics, J. Fluid Mech. 758, 508 (2014).

[6] J. W. M. Bush and I. Eames, Fluid displacement by high Reynolds number bubble motion in a thin gap, Intl. J. Multiphase Flow 24, 411 (1998).

[7] I. Eames and J. W. M. Bush, Longitudinal dispersion by bodies fixed in a potential flow, Proc. R. Soc. London 455, 3665 (1999).

[8] E. Bouche, S. Cazin, V. Roig, and F. Risso, Mixing in a swarm of bubbles rising in a confined cell measured by mean of PLIF with two different dyes, Exp. Fluids 54, 1552 (2013).

[9] E. Alméras, S. Cazin, F. Risso, V. Roig, S. Cazin, C. Plais, and F. Augier, Time-resolved measurement of concentration fluctuations in a confined bubbly flow by LIF, Int. J. Multiphase Flow 83, 153 (2016).

[10] J. Schmidt, R. Nassar, and A. Luebbert, Influence of the wakes in bubble driven multiphase flow systems, Chem. Eng. Sci. 47, 2295 (1992).

[11] J. Schmidt, R. Nassar, and A. Luebbert, Local dispersion in the liquid phase of gas-liquid reactors, Chem. Eng. Sci. 47, 3363 (1992).

[12] R. Nassar, J. Schmidt, and A. Luebbert, A stochastic dispersion model in gas-liquid flow systems, Chem. Eng. Sci. 47, 3657 (1992). 
[13] E. Bouche, V. Roig, F. Risso, and A.-M. Billet, Homogeneous swarm of high-Reynolds-number bubbles rising within a thin gap. Part 1. Bubble dynamics, J. Fluid Mech. 704, 211 (2012).

[14] V. Roig, M. Roudet, F. Risso, and A.-M. Billet, Dynamics of a high-Reynolds-number bubble rising within a thin gap, J. Fluid Mech. 707, 444 (2012).

[15] A. Filella, P. Ern, and V. Roig, Oscillatory motion and wake of a bubble rising in a thin-gap cell, J. Fluid Mech. 778, 60 (2015).

[16] F. Risso, Theoretical model for $k^{-3}$ spectra in dispersed multiphase flow, Phys. Fluids 23, 011701 (2011).

[17] F. Risso, Physical interpretation of probability density functions of bubble-induced agitation, J. Fluid Mech. 809, 240 (2016).

[18] E. Alméras, Propriétés de transport et de mélange dans les écoulements à bulles, Ph.D. thesis, Institut Nationale Polytechnique de Toulouse (2011).

[19] A. Loisy, A. Naso, and P. D. M. Spelt, The effective diffusivity of ordered and freely evolving bubbly suspensions, J. Fluid Mech. 840, 215 (2018). 